\title{
Bangla to English Code Switching in Bangladeshi Commercial Cinema \\ NASREEN SULTANA
}

\begin{abstract}
The language of media, especially language of cinema is relatively an unexplored area in the field of academic research. In Bangladeshi commercial cinema, it is often noticed that actors switch their codes from Bangla to English while speaking. However, it should not be thought that in cinema code switching is done without any reason; rather code switching in the dialogues is designed to convey the intended meaning. Therefore, it is important to investigate the phenomenon of code switching in Bangladeshi commercial cinema from an academic point of view. This research is descriptive and qualitative in nature. Its purpose is to find out the reasons and types of code switching which are found in the commercial films of Bangladesh. For this research, data are collected from commercial Bangladeshi films and interviews have been conducted with experts from linguistics and film background. After collecting the data, they are transcribed, tabulated and analyzed in terms of the research objectives.
\end{abstract}

\section{Introduction}

In the early 1920s cinema achieved a rivalry position against theatre and opera houses because of its glamour as well as grandeur and by the end of silent period cinema had established itself not only as an industry but also as the 'seventh art' (Smith, 1996, p. 3) due to incorporation of verbal dialogues. Quder (1993) calls this the culmination period in the history of motion pictures (p. 11). Cinema became the most important form of art in the entertainment world and later became the most important media. Adding verbal sound is an important phenomenon in cine history, which is the key reason behind the huge success of cinema. Smith (1996) names this event as a 'revolution' in the cine history, since adding 'synchronized 
sound dialogue' changed the cine art form into something else which it was never before (p. 207).

At present dialogue or verbal language is an integral part of films and due to globalization, usage of various languages or codes in films is very common to mass audience. Nowadays switching codes from one language to another is noticed in numerous films irrespective of countries. In modern cinema, practice of various languages is a widespread matter and code switching, in fact, makes the content more interesting.

Following the rest of the world, the tendency of code switching is prominent also in the cinemas of South-Asian countries such as Bangladesh, India, Malaysia, Pakistan and so on. In case of Bangladesh, frequent switching of code from Bangla to English is noticed in the commercial cinema. However, it should not be thought that Bangla to English code switching in Bangladeshi commercial cinema is done abruptly; rather there are some specific functions of code switching in cinema, which make the cine language more attention-grabbing, remarkable and meaningful.

\section{Problem Statement}

In Bangladeshi cinema there is much to explore about the language of cinema, since it is important and interesting and hence, demands a formal research to know more about it. This study attempts to show the function and type of Bangla to English switching found in Bangladeshi commercial cinema in various ways, which has been remained unidentified so far.

\section{Research Objectives}

The central research objectives are:

1) To find out the types of code switching used in commercial Bangladeshi cinemas. 
2) To find out the reasons of code switching in Bangladeshi commercial cinema.

\section{Research design}

The research is descriptive, non-experimental and investigative in nature. It attempts to explore the nature of code switching found in Bangladeshi commercial cinema. Its purpose is to look at the nature of code switching in the commercial cinemas of Bangladesh. The analysis of the data aims to provide answer to the central research objectives.

\section{Sampling}

The sampling is a two prong approach: one is textual and another is oral. The textual sampling is made up of 33 (thirty three) selected Bangladeshi movies and the oral sampling comprises of nine academicians from the discipline of linguistics and media.

\section{Data Collection and Analysis Procedure}

Films are selected randomly from 1970-2009 for the purpose of collecting data. Later, some experts have been interviewed both in formal and informal setting. After collecting the data, they are transcribed and tabulated. Information is compiled, tabulated and is analyzed by both qualitatively and quantitatively.

\section{Operational definitions}

The operational definitions of the research are given below:

\section{Commercial cinema}

Commercial cinemas are often termed as 'films of mass consumption' (Armburst, 1995, p. 82), that is, these films are made for common people based on their interest. Armburst believes that commercial cinema might not be intellectual but they are what the masses like. They establish convention among people, which is closely tied with the prevailing culture, 
ideology and language of a country (p. 117). However, Farid (1988) makes a harsh comment labeling commercial cinema 'for merchant and middleman' (p. 18), whose intention is only to do business, but he accepts the fact that one can see the social and political scenario of a society only in commercial cinema. Nasreen and Haque (as cited in Shahaduzzaman, 2008, p. 71) term the commercial cinema as 'dorshok ki khai' (what public eats), since it reflects the thought, hope and desire of the majority in the society. It can be understood that commercial cinema means the main stream films of a country which reflect the society and its people and are made for mass audience.

\section{Code switching/Code mixing}

Speakers keep switching to various styles, registers or dialects while speaking and this is called code switching. Gumperz (1982, p. 159) and Wardhaugh (1992, p. 103) both state that in code switching two languages are juxtaposed within the same speech exchange and it goes randomly. So, code switching means any kind of language shift in the speech. Meyerhoff (2006) believes that people who have command on more than one variety and are aware about the context usually use code switching (p. 116).

However, there is a controversy in between code switching and code mixing, since some researchers do not agree that code switching and mixing are same. Meyerhoff (2006) comments that code mixing is a 'cover term' for code switching and so there is no need to use separate terminology to identify them separately (p. 116). Nonetheless, generally it is thought that code switching occurs across the borders and code mixing occurs within the sentence, but there are not many differences between them. Therefore, code switching and mixing can be used interchangeably. 


\section{Literature Review}

\section{Language in Bangladeshi Cinema}

Very little writing has been found on Bangladeshi films and almost no writing is available on the language of Bangladeshi cinema except the historical writing of Kabir's Film in Bangladesh (1979). He actually realizes the importance of language in Bangladeshi cinema and therefore comments, "it could not be a matter of happiness for anyone having faith in the potential of the cinema in Bangladesh to realize that even after decade of film production true language of films art has yet to make its confident appearance in the arena" (p. 92). For Kabir (1979, p. 96), language of film art is 'plastic' in nature which is capable of expressing the most difficult thoughts explicitly. Later, he emphasizes on dialogues saying that dialogues are important to express the messages properly:

A correct screenplay will never allow audience interest to stray away by overloading the screenplay with dispensable details...other essential elements such as dialogues, music, incidental sound effects etc. must play a secondary role in Bangladeshi cinema...in other words, dialogues would come to visual's rescue only when latter's power of communication will appear inadequate . (p. 96)

\section{Code Switching in Cinema}

Armburst's (1995) investigation on code switching in cinema demonstrates that the language of films is not exclusively verbal, but consists of images coupled with verbal expression in a narrative framework and there are a number of codes evident in films, which are not necessarily dependent strictly on verbal expression. Armburst (1995) provides some characteristics of code switching in cinema. They are:

- Code switching in cinema suggests that both the filmmaker and the audience are familiar with both codes and this indicates a kind of identification in between the audience and the characters in the films. 
Therefore to understand such kind of code switching exposure towards education is not needed. However, sometimes the occurrence of foreign words might create a gap between the film and the audience. So code switching has to be very sophisticated and careful.

- Usually English is the common verbal code switching in cinema.

- Usually the effect of switching is either comic or sinister.

- Code switching is used to create sensation among the viewers.

- Sometimes code switching may be used in films to show a kind of solidarity to a particular culture or language.

- In cinema rich people show a reach variety of code switching to establish their upper position. (p. 98-115)

In Bangladeshi cinema also, actors use different variation of languages especially Bangla to English code switching. Sometimes it is done even unconsciously. Thus it has become an integral part of cine language.

Switching of codes is always common in cinema. In this research, the purpose is to reveal the nature of code switching found in the commercial Bangla cinema.

\section{Types of code switching}

Many researchers have identified three types of code switching, such as, tag switching, inter sentential and intra sentential (Poplack, 1980; Wardaugh, 1992; Holmes, 1992; and Romaine, 1995). They are given below as Poplack (1980) has described:

\section{Tag switching}

It is an insertion of a tag from one language into the utterance of another language. Since tags have minimal syntactic restrictions, it can be easily inserted in an utterance without violating the syntactic rules. Hence, even 
monolingual speakers can easily use tag switching, such as, you know, I mean are the most common phrases of English language which are frequently used in other languages in a form of tag switching.

\section{Inter-sentential}

Here switching occurs in the sentence boundary. It is generally thought that this type of switching needs good fluency in both the languages, since the speaker needs to maintain the rules of both languages which he uses in the switching.

\section{Intra-sentential}

Here code-switching means different types of switching occur within the sentence or clause boundary. Generally this type of switching needs mastery in both the languages since the speakers need to use different forms of switching within a single discourse. However, it is noticed that sometimes inefficient speakers also tend to initiate this sort of switching. In this case, speakers are used to inserting a noun phrase because inserting a noun phrase does not need a high syntactic mastery and thereof speakers find it easy to insert a noun phrase in the sentence.

\section{Discussion and findings}

After watching the movies, the data has been tabulated (Table-1). Table 1 shows the reasons and types of code switching which are usually found in the movies. Collected data from the interview are tabulated and analyzed in Table 2, where the experts has given their opinions about the reasons of code switching in the commercial films of Bangladesh.

\section{Reasons of code switching}

According to Table 1, there are some important reasons behind code switching in Bangladeshi commercial cinema. The table is given below followed by a discussion: 
Table 1: Reasons, examples and classifications of code switching found in Bangladesh commercial cinema from 1970 to 2009

\begin{tabular}{|c|c|c|c|c|c|}
\hline \multirow{2}{*}{$\begin{array}{l}\text { Name of } \\
\text { the Movies } \\
\text { (Year of } \\
\text { release) }\end{array}$} & \multirow{2}{*}{$\begin{array}{l}\text { Language } \\
\text { involved } \\
\text { in code } \\
\text { switching }\end{array}$} & \multirow{2}{*}{$\begin{array}{l}\text { Reasons } \\
\text { behind code } \\
\text { switching in } \\
\text { Bangladeshi } \\
\text { commercial } \\
\text { cinemas }\end{array}$} & \multicolumn{3}{|c|}{$\begin{array}{l}\text { Types of code switching found in cinema with } \\
\text { examples }\end{array}$} \\
\hline & & & Tag switching & Inter sentential & Intra sentential \\
\hline $\begin{array}{l}\text { 1. Taka Ana } \\
\text { Pai (1973) }\end{array}$ & $\begin{array}{l}\text { Bangla } \\
\text { English } \\
\text { Hindi }\end{array}$ & $\begin{array}{l}\text { Habituation } \\
\text { To show } \\
\text { high status }\end{array}$ & $\begin{array}{l}\text { Ore baba, } \\
\text { amake lecture } \\
\text { dei. } \\
\text { Ha } \\
\text { appointment to } \\
\text { kore e eshesi. } \\
\text { Ekkebare good } \\
\text { boy re }\end{array}$ & & \\
\hline $\begin{array}{l}\text { 2. Beiman } \\
(1974)\end{array}$ & & $\begin{array}{l}\text { To show } \\
\text { highly } \\
\text { educated } \\
\text { To show } \\
\text { status } \\
\text { Habituation }\end{array}$ & $\begin{array}{l}\text { Right apanar } \\
\text { prostab ami } \\
\text { manlam. } \\
\text { Montu k tu } \\
\text { capture kore } \\
\text { rakish... bye } \\
\text { bye }\end{array}$ & & $\begin{array}{l}\text { It is so nice Naj j } \\
\text { tumi amar } \\
\text { shathe berate } \\
\text { jabe? Oh! Sure } \\
\text { lets go. } \\
\text { Please aunty } \\
\text { othona...o my } \\
\text { sweet darling } \\
\text { Naj, tumi jege } \\
\text { aso? }\end{array}$ \\
\hline $\begin{array}{l}\text { 3. Mon Jare } \\
\text { Chai (1976) }\end{array}$ & $\begin{array}{l}\text { Bangla } \\
\text { English }\end{array}$ & $\begin{array}{l}\text { To raise } \\
\text { humor/com } \\
\text { edy } \\
\text { Habitation }\end{array}$ & $\begin{array}{l}\text { Are era to } \\
\text { international } \\
\text { chor }\end{array}$ & $\begin{array}{l}\text { Such a bad } \\
\text { situation, ekhon } \\
\text { ki hobe? }\end{array}$ & \\
\hline $\begin{array}{l}\text { 4. Jay } \\
\text { Parjay } \\
(1976)\end{array}$ & & $\begin{array}{l}\text { Habituation } \\
\text { To maintain } \\
\text { official } \\
\text { norms }\end{array}$ & $\begin{array}{l}\text { Entry fee } \\
\text { ekshototo } \\
\text { taka } \\
\text { Next, cholo } \\
\text { next heroine. } \\
\text { Don't forget j } \\
\text { eta ekta police } \\
\text { station. }\end{array}$ & & \\
\hline $\begin{array}{l}\text { 5. Gangchil } \\
(1980)\end{array}$ & $\begin{array}{l}\text { Bangla } \\
\text { English }\end{array}$ & $\begin{array}{l}\text { To show } \\
\text { being } \\
\text { educated }\end{array}$ & $\begin{array}{l}\text { Chalun na ek } \\
\text { cup coffee hoe } \\
\text { jak!! }\end{array}$ & $\begin{array}{l}\text { Chalun na ek } \\
\text { cup coffee hoe } \\
\text { jak!!/ I would be }\end{array}$ & \\
\hline
\end{tabular}




\begin{tabular}{|c|c|c|c|c|c|}
\hline & & $\begin{array}{l}\text { To show } \\
\text { high status } \\
\text { Habituation }\end{array}$ & & $\begin{array}{l}\text { pleased } \\
\text { My chief } \\
\text { engineer, Nishad } \\
\text { eke kaj kormo } \\
\text { bujie dao, I think } \\
\text { he will be } \\
\text { interested. }\end{array}$ & \\
\hline $\begin{array}{l}\text { 6. Nagin } \\
\text { (1980) }\end{array}$ & N/A & & 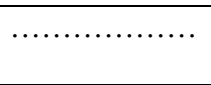 & 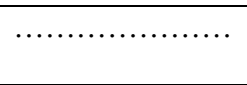 & ……............. \\
\hline $\begin{array}{l}\text { 7. Gaddar } \\
\text { (1983) }\end{array}$ & $\begin{array}{l}\text { Bangla } \\
\text { English } \\
\text { Arabic }\end{array}$ & $\begin{array}{l}\text { To show } \\
\text { authority } \\
\text { To show } \\
\text { respect } \\
\text { according to } \\
\text { religion } \\
\text { Habituation }\end{array}$ & $\begin{array}{l}\text { Asha kori I } \\
\text { am clear./ Ami } \\
\text { bolesilam } \\
\text { na/Thank you } \\
\text { very much }\end{array}$ & & \\
\hline $\begin{array}{l}\text { 8. Ghar } \\
\text { Bhanga } \\
\text { Ghar (1985) }\end{array}$ & N/A & …........... & & & \\
\hline 9. Daetto & $\begin{array}{l}\text { Bangla } \\
\text { English }\end{array}$ & $\begin{array}{l}\text { Habituation } \\
\text { To Maintain } \\
\text { official/situa } \\
\text { tional norms }\end{array}$ & $\begin{array}{l}\text { Notun k die } \\
\text { heroin er part } \\
\text { koraben? } \\
\text { /chobi } \\
\text { superhit. } \\
\text { Film office e } \\
\text { koto e chance } \\
\text { r jonno ashe. } \\
\text { Congratualatio } \\
\text { n./thank } \\
\text { you... } \\
\text { hateleder } \\
\text { hato norom }\end{array}$ & & \\
\hline $\begin{array}{l}10 . \\
\text { Opekkha } \\
\text { (1987) }\end{array}$ & $\begin{array}{l}\text { Bangla } \\
\text { English }\end{array}$ & $\begin{array}{l}\text { To create } \\
\text { humor } \\
\text { To put } \\
\text { emphasis } \\
\text { Habituation }\end{array}$ & $\begin{array}{l}\text { Very bad very } \\
\text { bad, meyeder } \\
\text { shathe emon } \\
\text { korena/ } \\
\text { rubbish, dile } \\
\text { to practice ta } \\
\text { mati kore!! }\end{array}$ & & \\
\hline $\begin{array}{l}\text { 11. Tin } \\
\text { Konna } \\
\text { (1989) }\end{array}$ & $\begin{array}{l}\text { Bangla } \\
\text { English }\end{array}$ & $\begin{array}{l}\text { To create } \\
\text { humor } \\
\text { To maintain } \\
\text { official } \\
\text { norms } \\
\text { Habituation } \\
\end{array}$ & $\begin{array}{l}\text { Amar darling } \\
\text { re...yahooh. } \\
\text { darling tumi } \\
\text { amar } \\
\text { hoibana...to } \\
\text { mare ami kiss }\end{array}$ & $\begin{array}{l}\text { May I come in } \\
\text { sir? Oh yes...ei } \\
\text { hoche Miss. } \\
\text { Roji./amra } \\
\text { porichito/ OH! } \\
\text { That's fine. Miss. }\end{array}$ & \\
\hline
\end{tabular}




\begin{tabular}{|c|c|c|c|c|}
\hline & & & dimu & $\begin{array}{l}\text { Kobita tomake } \\
\text { shahaijo korbe/ } \\
\text { Yes sir. }\end{array}$ \\
\hline $\begin{array}{l}12 . \\
\text { Shuvoda } \\
\text { (1987) }\end{array}$ & N/A & ................. & 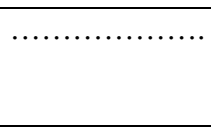 & \\
\hline $\begin{array}{l}\text { 13. Bashona } \\
\text { (1989) }\end{array}$ & $\begin{array}{l}\text { Bangla } \\
\text { English }\end{array}$ & $\begin{array}{l}\text { Habituation } \\
\text { To Maintain } \\
\text { official/situa } \\
\text { tional norms } \\
\text { To raise } \\
\text { humor }\end{array}$ & $\begin{array}{l}\text { Hego } \\
\text { understanding } \\
\text { hoia gesega. } \\
\text { Temon } \\
\text { kisuna- minor } \\
\text { injury, she will } \\
\text { be alright. }\end{array}$ & $\begin{array}{l}\text { Temon kisuna- } \\
\text { minor injuri, she } \\
\text { will be alright. }\end{array}$ \\
\hline $\begin{array}{l}14 . \\
\text { Okritoggo } \\
(1990)\end{array}$ & $\begin{array}{l}\text { Bangla } \\
\text { English }\end{array}$ & $\begin{array}{l}\text { Habituation } \\
\text { To Maintain } \\
\text { official/situa } \\
\text { tional norms }\end{array}$ & $\begin{array}{l}\text { Ladies sata r } \\
\text { niche seleder } \\
\text { matha } \\
\text { dukano } \\
\text { thikna } \\
\text { Sir, Madam } \\
\text { apnake call } \\
\text { koresen/May } \\
\text { I come in } \\
\text { madam, Ha } \\
\text { ashun }\end{array}$ & \\
\hline $\begin{array}{l}\text { 15. Den } \\
\text { Mohor }\end{array}$ & $\begin{array}{l}\text { Bangla } \\
\text { Hindi }\end{array}$ & $\begin{array}{l}\text { To show } \\
\text { high status } \\
\text { To show } \\
\text { respect } \\
\text { according to } \\
\text { religion }\end{array}$ & $\begin{array}{l}\text { Apni amader } \\
\text { jobon noshto } \\
\text { korben na, } \\
\text { Allah hafez } \\
\text { Beta Ajmol, } \\
\text { raaj } \\
\text { shobsomoi } \\
\text { mathai rakte } \\
\text { hoi }\end{array}$ & \\
\hline $\begin{array}{l}\text { 16. Chaowa } \\
\text { Theke } \\
\text { Paowa }\end{array}$ & $\begin{array}{l}\text { Bangla } \\
\text { English }\end{array}$ & $\begin{array}{l}\text { To show } \\
\text { educated } \\
\text { To show } \\
\text { high status } \\
\text { Habituation }\end{array}$ & $\begin{array}{l}\text { Ekta } \\
\text { cha/Thank } \\
\text { you } \\
\text { Bah! } \\
\text { Excellent..cmo } \\
\text { takar...wow } \\
\text {...that's it. } \\
\text { Sire felo...go } \\
\text { ahead }\end{array}$ & \\
\hline $\begin{array}{l}\text { 17. Tomake } \\
\text { Chai }\end{array}$ & $\begin{array}{l}\text { Bangla } \\
\text { English }\end{array}$ & $\begin{array}{l}\text { To show } \\
\text { respect }\end{array}$ & $\begin{array}{l}\text { Amar money- } \\
\text { bag ta khali }\end{array}$ & \\
\hline
\end{tabular}




\begin{tabular}{|c|c|c|c|c|c|}
\hline & Arabic & $\begin{array}{l}\text { according to } \\
\text { religion } \\
\text { Habituation }\end{array}$ & $\begin{array}{l}\text { hoe gese } \\
\text { O apni e } \\
\text { regular } \\
\text { customer/ } \\
\text { ekhon apni e } \\
\text { ice-cream } \\
\text { khan/ thank } \\
\text { you }\end{array}$ & & \\
\hline $\begin{array}{l}\text { 18. Ei Ghar, } \\
\text { Ei Shanshar }\end{array}$ & $\begin{array}{l}\text { Bangla } \\
\text { Hindi } \\
\text { English } \\
\text { Arabic }\end{array}$ & $\begin{array}{l}\text { Habituation } \\
\text { To maintain } \\
\text { the } \\
\text { situational } \\
\text { norms } \\
\text { To create } \\
\text { humor } \\
\text { To show } \\
\text { respect } \\
\text { according to } \\
\text { religion }\end{array}$ & $\begin{array}{l}\text { College e } \\
\text { shobai } \\
\text { friends...sorry } \\
\text { bole de... } \\
\text { Bad luck } \\
\text { apnar meye } \\
\text { chole } \\
\text { gelo...okay } \\
\text { goodbye } \\
\text { Ami nijer risk } \\
\text { e or nam e } \\
\text { loan dite } \\
\text { pari/thank } \\
\text { you uncle, } \\
\text { thank you. }\end{array}$ & $\begin{array}{l}\text { May I come in } \\
\text { sir?/ eto deri } \\
\text { keno, why so } \\
\text { late? }\end{array}$ & \\
\hline $\begin{array}{l}\text { 19. Jibon } \\
\text { Shanshar }\end{array}$ & $\begin{array}{l}\text { Bangla } \\
\text { English } \\
\text { Arabic }\end{array}$ & $\begin{array}{l}\text { To show } \\
\text { respect } \\
\text { according to } \\
\text { religion } \\
\text { Habituation } \\
\text { To maintain } \\
\text { the official } \\
\text { norms } \\
\text { To put } \\
\text { emphasis }\end{array}$ & $\begin{array}{l}\text { Ashte pari/o } \\
\text { yes yes / sit } \\
\text { down, tumi e } \\
\text { to first } \\
\text { hoeso/ thank } \\
\text { you sir. }\end{array}$ & $\begin{array}{l}\text { Ami e jobon } \\
\text { grina kori...I } \\
\text { hate this life..I } \\
\text { hate this life }\end{array}$ & \\
\hline $\begin{array}{l}\text { 20. Shudu } \\
\text { Tumi }\end{array}$ & $\begin{array}{l}\text { Bangla } \\
\text { English } \\
\text { Arabic }\end{array}$ & $\begin{array}{l}\text { To show } \\
\text { respect } \\
\text { according to } \\
\text { religion } \\
\text { Habituation } \\
\text { To show } \\
\text { high status }\end{array}$ & $\begin{array}{l}\text { Ei follow me/ } \\
\text { shut up, maro } \\
\text { oke. }\end{array}$ & & \\
\hline $\begin{array}{l}\text { 21. Ontore } \\
\text { Ontore }\end{array}$ & $\begin{array}{l}\text { Bangla } \\
\text { English }\end{array}$ & $\begin{array}{l}\text { To put } \\
\text { emphasis } \\
\text { To show } \\
\text { high status } \\
\text { To show }\end{array}$ & $\begin{array}{l}\text { Ki } \\
\text { shundor...bea } \\
\text { utiful...owo... } \\
\text { onek shundor } \\
\text { jaiga }\end{array}$ & $\begin{array}{l}\text { Beg your } \\
\text { pardon...forgive } \\
\text {...onugroho } \\
\text { kore amake } \\
\text { khoma kore dao }\end{array}$ & \\
\hline
\end{tabular}




\begin{tabular}{|c|c|c|c|c|c|}
\hline & & $\begin{array}{l}\text { education } \\
\text { Habituation }\end{array}$ & & & \\
\hline $\begin{array}{l}\text { 22. Shopner } \\
\text { Nayok }\end{array}$ & $\begin{array}{l}\text { Bangla } \\
\text { English }\end{array}$ & $\begin{array}{l}\text { To maintain } \\
\text { office } \\
\text { environment } \\
\text { To show } \\
\text { education } \\
\text { To show } \\
\text { high status } \\
\text { Habituation }\end{array}$ & $\begin{array}{l}\text { Hey, young } \\
\text { man, nam } \\
\text { ki?/ public } \\
\text { Rag korona, } \\
\text { fun korsilam }\end{array}$ & & \\
\hline $\begin{array}{l}\text { 23. Voinkor } \\
\text { Raja }\end{array}$ & $\begin{array}{l}\text { Bangla } \\
\text { English }\end{array}$ & Habituation & $\begin{array}{l}\text { Kothin shajar } \\
\text { bebostha } \\
\text { korben/ yes } \\
\text { sir. } \\
\text { Motive khuje } \\
\text { ber korar } \\
\text { daetto police r } \\
\text { Ei light, } \\
\text { camera shob } \\
\text { ready? Heroine } \\
\text { ki ready } \\
\text { hoise? Madam } \\
\text { koi }\end{array}$ & $\begin{array}{l}\text { This is the last } \\
\text { warning/sir apni } \\
\text { amake vul } \\
\text { bujsen/what do } \\
\text { you mean, } \\
\text { amake ja bujabe } \\
\text { ami bujbo, am I } \\
\text { a foolish guy? } \\
\text { Stop it Ana, stop } \\
\text { it, what the hell } \\
\text { you are talking } \\
\text { about? }\end{array}$ & \\
\hline $\begin{array}{l}\text { 24. Jamidar } \\
\text { Barir Meye }\end{array}$ & $\begin{array}{l}\text { Bangla } \\
\text { English }\end{array}$ & $\begin{array}{l}\text { To show } \\
\text { high status } \\
\text { To maintain } \\
\text { certain } \\
\text { situational } \\
\text { norms } \\
\text { Habituation }\end{array}$ & $\begin{array}{l}\text { Bhaia amake } \\
\text { shundor } \\
\text { lagesena? You } \\
\text { are looking } \\
\text { wonderful. } \\
\text { Shotti?? } \\
\text { Hundred } \\
\text { percent shotti }\end{array}$ & $\begin{array}{l}\text { Ladies and } \\
\text { gentlemen, ejker } \\
\text { ei party te asher } \\
\text { jonno shobai ke } \\
\text { donnobad. } \\
\text { O Hasi lets } \\
\text { go...come }\end{array}$ & \\
\hline $\begin{array}{l}\text { 25. Estrir } \\
\text { Morjada }\end{array}$ & $\begin{array}{l}\text { Bangla } \\
\text { English } \\
\text { Arabic } \\
\text { Hindi }\end{array}$ & $\begin{array}{l}\text { To maintain } \\
\text { official } \\
\text { jargon/ } \\
\text { norms/ } \\
\text { situation } \\
\text { Habituation } \\
\text { / social norm } \\
\text { To show } \\
\text { respect } \\
\text { according to } \\
\text { religion }\end{array}$ & $\begin{array}{l}\text { Operation na } \\
\text { korel patient } \mathrm{r} \\
\text { khoti hoe jabe } \\
\text { Valo ase Sir. } \\
\text { Good, very } \\
\text { good. Next } \\
\text { tomake ekta } \\
\text { operation e jete } \\
\text { hobe. } \\
\text { Hey rahmanur } \\
\text { rahim, tumi ei } \\
\text { dil-doria }\end{array}$ & & \\
\hline
\end{tabular}




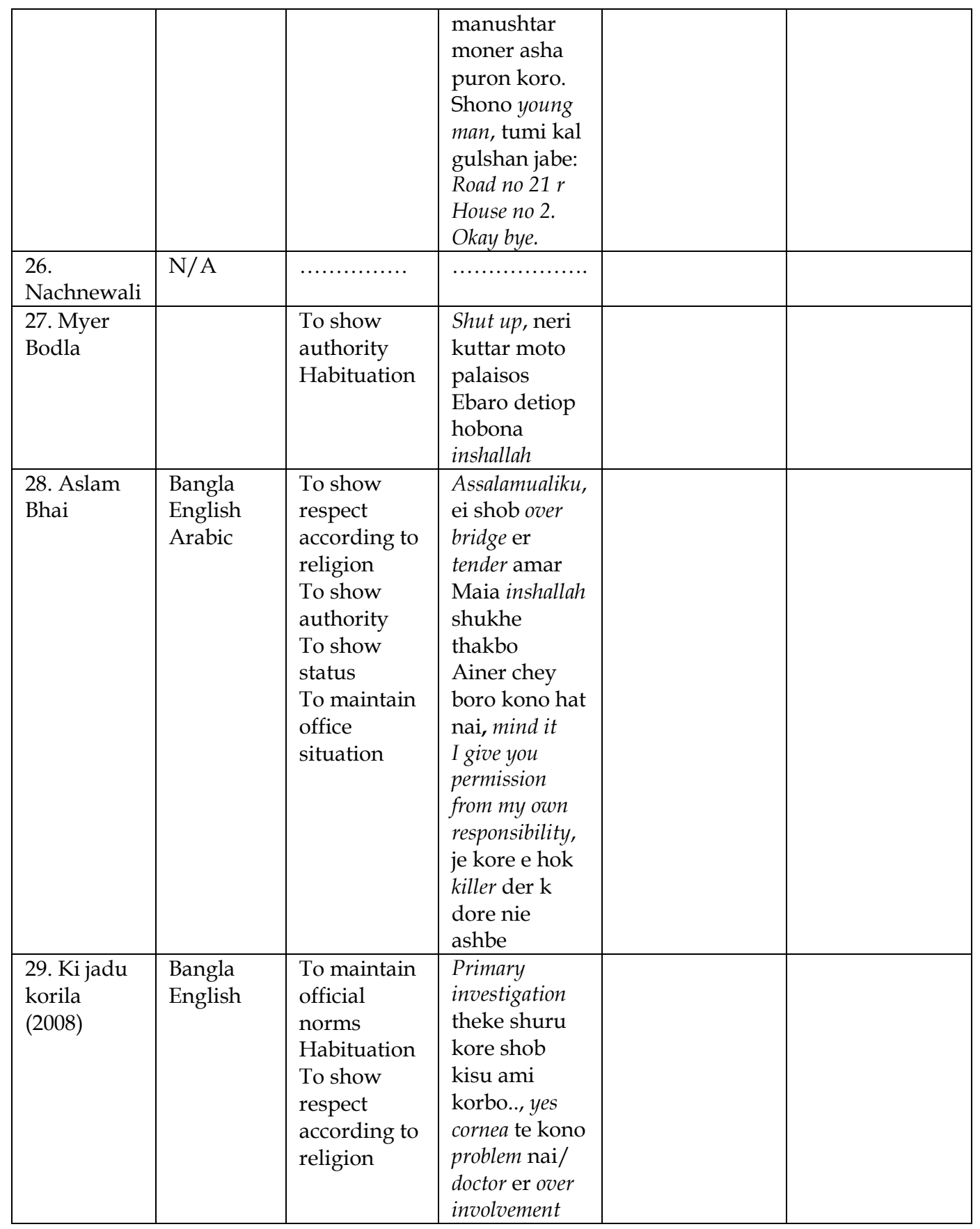




\begin{tabular}{|c|c|c|c|c|c|}
\hline & & & $\begin{array}{l}\text { patient } \mathrm{r} \\
\text { khotir karon } \\
\text { hote pare. }\end{array}$ & & \\
\hline $\begin{array}{l}\text { 30. Tomar } \\
\text { Jonno Pagol } \\
(2008)\end{array}$ & $\begin{array}{l}\text { Bangla } \\
\text { English } \\
\text { Arabic }\end{array}$ & Habituation & $\begin{array}{l}\text { Shomane goal } \\
\text { ditase apnare } \\
\text { Jiboner flight } \\
\text { raat dosh tai }\end{array}$ & & \\
\hline $\begin{array}{l}\text { 31. Dadima } \\
(2008)\end{array}$ & $\begin{array}{l}\text { Bangla } \\
\text { English } \\
\text { Hindi }\end{array}$ & $\begin{array}{l}\text { Express } \\
\text { anger } \\
\text { Habituation } \\
\text { of tagging } \\
\text { To show } \\
\text { high status } \\
\text { To show } \\
\text { education }\end{array}$ & $\begin{array}{l}\text { Amar Akash } \\
\text { ki mistake } \\
\text { korese }\end{array}$ & $\begin{array}{l}\text { Bye Prity, really } \\
\text { you are so pretty, } \\
\text { ha dost shotti } \\
\text { she is bold and } \\
\text { beautiful }\end{array}$ & $\begin{array}{l}\text { Amar kase } \\
\text { tucho, an old } \\
\text { haggard like you } \\
\text { Ke receive korbe } \\
\text { take, okay bye; } \\
\text { Hey man, hi } \\
\text { Akash }\end{array}$ \\
\hline $\begin{array}{l}\text { 32. Kopal } \\
(2009)\end{array}$ & $\begin{array}{l}\text { Bangla } \\
\text { English }\end{array}$ & $\begin{array}{l}\text { Habituation } \\
\text { To show } \\
\text { education } \\
\text { To put } \\
\text { emphasis }\end{array}$ & $\begin{array}{l}\text { Classroom e } \\
\text { dushtami } \\
\text { korse tai } \\
\text { teacher boka } \\
\text { dise } \\
\text { Chalo, let's go }\end{array}$ & & \\
\hline $\begin{array}{l}\text { 33. Kothin } \\
\text { Prem (2009) }\end{array}$ & $\begin{array}{l}\text { Bangla } \\
\text { English }\end{array}$ & $\begin{array}{l}\text { To put } \\
\text { emphasis } \\
\text { To show } \\
\text { anger }\end{array}$ & $\begin{array}{l}\text { Amar jonno } \\
\text { right } \mathrm{r} \text { wrong } \\
\text { shudu tumi. }\end{array}$ & $\begin{array}{l}\text { I love you, ami } \\
\text { shudu tomake } \\
\text { valobashi } \\
\text { O ami to kisu e } \\
\text { buhte parsina, } \\
\text { what's going here }\end{array}$ & \\
\hline
\end{tabular}

\section{Habituation}

From the analysis, it is found that in films code switching occurs as a result of mere habituation of the speakers. Primarily, English is used in switching the codes from Bangla to show the characters are educated. It is observed that Most of the characters are comfortable in mixing Bangla-English while conversing, so it does not seem artificial or imposed. Usually filler words, tag words, common English nouns or words are used in mixing the code. 


\section{To maintain situational norms}

Often code switching is done to maintain some kind of official or situational norms. Table-1 shows that sometimes the characters switch their codes from Bangla to English because situation demands. In office or business meeting, English phrases or jargons are used to maintain the official environment. Some common medical or official terms are used in many occasions to cope up the situation.

In Bangladesh, practice of English is common in official or formal situation. Sometimes, it is necessary to use English word to maintain the situation in places such as offices, universities or Hospitals. On the other hand, commercial cinema is the reproduction of real life into reel life. For this reason, in cellular world also the actors do some situational code switching imitating the authentic situational code switching to create a simulation of real life.

\section{To show high status or to create social distance}

In films often English words are used as a symbol of high status or to create social distance. When the characters are rich and educated, they intentionally switch their codes from Bangla to English to show high status, which creates a social distance between the speakers. Thus code switching is used to showcase the high status.

In fact in Bangladesh, the ability of speaking in English is thought to be a symbol of being rich or educated. Therefore, even in cinema also the characters tend to use English words as a part of their status symbol. However, sometimes this kind of code switching may sound artificial because of the unnecessary insertion and mispronunciation of some English words.

\section{To show authority}

At times, code switching is used when the characters are in an authoritative position and want to establish their authority. Often 
sentence-level code switching takes place to show that the users have good proficiency in English. The characters deliberately switch their codes from Bangla to English to show their importance and authority in a situation.

Actually in Bangladesh English proficiency is highly valued. In offices and formal situations Bangladeshis often use English to show their authority. Even in cinema also this social norm is evident; characters tend to switch from Bangla to English to demonstrate their superiority.

\section{To show being educated}

After the analysis, it is found that educated characters frequently use English words and sentences in their speaking. When someone comes back from abroad after finishing the higher studies, he tends to insert small English sentences while speaking. In addition, sometimes they try to exercise English accent to prove their long practice in English. Again university graduate students are found to switch their codes from Bangla to English when they are in party or gossiping with friends.

In Bangladesh, even in real situations also students or educated common people randomly switch their codes from Bangla to English. Therefore it is accepted that educated people will use English language with Bangla frequently without any hesitation. Nevertheless, sometimes the insertion of English words seems to be imposed when the characters add English accent in their English, since in reality many educated Bangladeshi English speakers actually do not use English accent while speaking.

\section{To raise humor}

To create comic situation is another reason behind switching the codes in Bangladeshi cinema. Often actors switch their codes from Bangla to English to generate funny situations. In many commercial 
films, it is found that comedians pronounce English words in a weird accent which creates humor.

From the situation or switching, it is apparent that the characters are not proficient English speakers. They make code switching only to weave a funny situation. Sometimes, to insert a sense of sarcasm also Bangla to English switching takes place.

\section{To put emphasis/ to create importance}

Bangla to English Code switching occurs when someone wants to emphasize on a particular dialogue or expression. Basically English language is used to accomplish the desired meaning in the conversation. This code switching is done in two ways: the characters pronounce the same dialogue two times in English to put more emphasis; and the other is once the dialogue is said in Bangla and then again the same dialogue is said in English. Generally to convey a specific message to the other within the conversation this kind of code switching is done. In these situations, usually the users are educated, rich and authoritative.

\section{To show anger}

Bangla-English code mixing is done intentionally when the characters express their anger or frustration. In fact, in Bangladesh even the general mass educated people also switch their codes from Bangla to English when they want to express their anger.

These are the above mentioned reasons for what code switching generally occurs in Bangladeshi commercial cinema.

\section{Types of code switching}

From the analysis, three types of code switching (Table 1) are found in Bangladeshi movies. They are tag switching, inter sentential code switching and intra sentential code switching, which are discussed below: 


\section{- Tag switching}

The analysis of the data (Table 1) shows that tag switching is the chief tendency of switching. It is seen that habitually code switching is done in word level either to convey a particular message or to show regular habit of the users. Often this switching becomes too frequent that even the audiences also do not notice that the speakers are switching their codes from Bangla to English. It is notable that use of English language is limited within the common English words or fixed phrase pattern.

However the data reveals that tag switching is done by uneducated person to create comic situation by adding weird accent in their English words. On the other hand, educated persons use tag switching out of habituation or they want to convey some specific message.

\section{- Inter sentential code switching}

In Bangladeshi commercial cinema (Table 1) inter sentential code switching do not occur frequently. In fact, to switch the code within the sentence boundary, a little mastery of language is needed, since it has a high syntactical restriction. That is why, in Bangladeshi commercial cinema when the characters are shown highly educated; only then inter sentential code switching takes place in the conversation.

\section{- Intra sentential code switching}

This kind of code switching is rare in Bangladeshi commercial cinema and it happens seldom. In the sampling (Table 1), it is found that characters who have come back from abroad or are highly educated make intra sentential code switching. In fact intra sentential code switching needs high proficiency in both the languages and it has a high syntactic restriction as well. Though it is a word or phrase level code switching, here unlike tag switching the speakers need to have good knowledge of grammar and code switching. It happens within the 
sentence boundary and to insert a foreign code, the speakers should know the rules of code switching.

Actually, in commercial cinema code switching is done in a way that lay people can understand them and so the level of code switching is minimal. Therefore, intra sentential code switching is not frequent in Bangladeshi commercial films.

\section{Findings from the interview}

To know the opinions of the experts, some professionals from film and linguistics background have been interviewed (Appendix A). The interview data are tabulated and analyzed in Table 2 to know the general opinions, what the experts think about the phenomenon of code switching in Bangladeshi commercial cinema. The table 2 is shown onto next page:

\begin{tabular}{|l|l|l|}
\hline S/N & $\begin{array}{l}\text { Back ground information of } \\
\text { the interviewees }\end{array}$ & $\begin{array}{l}\text { Reasons for code switching } \\
\text { in Bangladeshi commercial } \\
\text { cinema }\end{array}$ \\
\hline 1 & $\begin{array}{l}\text { He is the assistant professor of } \\
\text { ELT and linguistics at East West } \\
\text { University. Interest includes } \\
\text { linguistic imperialism, } \\
\text { language planning and policy, } \\
\text { linguistic diversity and } \\
\text { varieties. }\end{array}$ & $\begin{array}{l}\text { Laymen and general people } \\
\text { of Bangladesh are habituated } \\
\text { to use some common English } \\
\text { words. Sometimes people } \\
\text { assume that using English } \\
\text { words might increase their } \\
\text { status. }\end{array}$ \\
\hline 2 & $\begin{array}{l}\text { He is an assistant professor of } \\
\text { literature and cultural studies at } \\
\text { East West University. His area } \\
\text { of interest includes capitalism, } \\
\text { power relation among the } \\
\text { countries and cultural variation } \\
\text { among countries }\end{array}$ & $\begin{array}{l}\text { Language is meant to be } \\
\text { changed and humans tend to } \\
\text { adapt new words which } \\
\text { seem to be convenient to } \\
\text { them. Because of capitalism } \\
\text { and globalization, now even } \\
\text { in cinema also foreign words } \\
\text { are being used. However, in } \\
\text { Bangladeshi cinema Bangla } \\
\text { to English code switching is } \\
\text { limited, since they tend to }\end{array}$ \\
\hline
\end{tabular}




\begin{tabular}{|l|l|l|}
\hline & use few English words. \\
\hline 3 & $\begin{array}{l}\text { She is a senior lecturer of } \\
\text { English literature in East West } \\
\text { University. Her area of interest } \\
\text { includes post colonial literature } \\
\text { and cultural studies. }\end{array}$ & $\begin{array}{l}\text { Basically code switching is } \\
\text { done to show the status or } \\
\text { education. Often code } \\
\text { switching seems to be } \\
\text { artificial and imposed. }\end{array}$ \\
\hline 4 & $\begin{array}{l}\text { He is an assistant professor of } \\
\text { film and media studies in } \\
\text { Stamford University } \\
\text { Bangladesh. He is interested in } \\
\text { the recent development of film } \\
\text { industry in Bangladesh. }\end{array}$ & $\begin{array}{l}\text { In Bangladeshi commercial } \\
\text { cinema code switching is } \\
\text { done because the same kind } \\
\text { of switching is done in the } \\
\text { real life also. Cinema shows } \\
\text { what is going on in the } \\
\text { society. Therefore in cinema } \\
\text { too the usage of English } \\
\text { words is common, however, } \\
\text { only the switching of phrases } \\
\text { are prominent. }\end{array}$ \\
\hline 7 & $\begin{array}{l}\text { He is a part time professor of } \\
\text { film and media studies in } \\
\text { Stamford University } \\
\text { Bangladesh. His research area } \\
\text { includes the comparative study } \\
\text { of world and Asian films, film } \\
\text { culture and its history. }\end{array}$ & $\begin{array}{l}\text { Basically Bangla to English } \\
\text { code switching occurs in } \\
\text { Bangladeshi cinema to show } \\
\text { that they are the part of } \\
\text { world culture of using } \\
\text { English language. Nowadays } \\
\text { English language usage has } \\
\text { become a common habit for } \\
\text { Bangladeshi people. }\end{array}$ \\
\hline 5 \\
$\begin{array}{l}\text { He is a senior lecturer of film } \\
\text { and media studies in Stamford } \\
\text { University Bangladesh. He is } \\
\text { anmera work of films } \\
\text { and media studies in Stamford } \\
\text { interested in the Montage of } \\
\text { films }\end{array}$ & $\begin{array}{l}\text { In Bangladeshi cinema, the } \\
\text { usage of English language is } \\
\text { a result of mere habituation. } \\
\text { But, usually only words or at } \\
\text { max phrases are used in } \\
\text { switching the codes. }\end{array}$ \\
\hline $\begin{array}{l}\text { Code switching occurs in } \\
\text { Bangla cinema, because it is } \\
\text { very much influenced by } \\
\text { Hindi cinema culture, where } \\
\text { English language is used } \\
\text { frequently. Nonetheless, }\end{array}$ \\
\hline
\end{tabular}




\begin{tabular}{|l|l|l|}
\hline 8 & $\begin{array}{l}\text { extensive use of English is } \\
\text { not prominent. }\end{array}$ \\
\hline 8 & $\begin{array}{l}\text { She is an assistant professor of } \\
\text { department of English in } \\
\text { Stamford University. Her area } \\
\text { of interest includes post } \\
\text { modernist studies and } \\
\text { feminism. }\end{array}$ & $\begin{array}{l}\text { Code switching happens in } \\
\text { Bangladeshi commercial } \\
\text { cinema as a result of } \\
\text { habituation of using English } \\
\text { language for a long time. }\end{array}$ \\
\hline $\begin{array}{l}\text { She is an assistant professor of } \\
\text { department of English in } \\
\text { Stamford University. Her area } \\
\text { of interest includes } \\
\text { sociolinguistics, ELT and world } \\
\text { language. }\end{array}$ & $\begin{array}{l}\text { To show that the character } \\
\text { are habituated in using } \\
\text { foreign languages like } \\
\text { English, Bangla-English code } \\
\text { switching is used. Generally, } \\
\text { in this case tag switching } \\
\text { seems to be popular in } \\
\text { cinema. }\end{array}$ \\
\hline
\end{tabular}

The findings from the interview are given below:

- Bangla to English code switching is present in Bangladeshi cinema because of globalization. Nowadays, mass people are open to the western culture. Therefore they want to imitate that culture. Accordingly Bangladeshi commercial cinema also tries to imitate that culture of using English words and phrases in the dialogues so that they can be in the flow of the society.

- English language has become an integral part of the society. Nowadays, English language is a marker of high status. Education and wealth. Therefore, in commercial cinema switching from Bangla to English occurs to depict the reality. Bangla to English code switching in the cinema is just the result of the habituation of using this language for a long time.

- Usually, small chunks are used to switch the codes from Bangla to English. However, educated or rich characters are found to use longer phrases or sentences of English as well. 


\section{General findings}

Apart from the research objectives also the researcher has found some general findings, such as:

1. Basically when the setting is rustic or rural there is minimal usage of code switching and sometimes almost no usage at all.

2. In many occasions, code switching seems to be artificial or imposed as the speakers tend to add accent with the English words and eventually they sound weird.

3. On the whole, Bangla to English code switching is used to show status, education and authority

4. Generally, Bangla to English code switching is done in such a way that even the lay man also can understand the meaning.

\section{Delimitation}

Each year different kinds of cinemas are released in Bangladesh, such as, documentary, arts, commercial, historical and many more; however, the researcher has chosen only the commercial movies for her study. Hence, the findings should not be generalized.

\section{References}

Armbrust, W. (1995). New Cinema, Commercial Cinema, and the Modernist Tradition in Egypt. Alif: Journal of Comparative Poetics, 15, 81-129.

Farid, S. (1988). Huwiyat al-Sinima al-'Arabiyya. Beirut: Dar al-Farabi, 12, 5-28.

Gumperz, J. (1982). Discourse Strategies. Cambridge: Cambridge University Press.

Holmes, J. (2001). An Introduction to Sociolinguistics ( $2^{\text {nd }}$ ed.). UK: Pearson Education Ltd.

Kabir, A. (1979). Film in Bangladesh. Dhaka: Bangla Academy. 
Meyerhoff, M. (2006). Introducing Linguistics. London: Rutledge.

Quder, T. M. (1993). Bangladesher Chalachitre Shilpo. Dhaka: Bangla Academy.

Poplack. S. (1980). Sometimes I will Start a Sentence in Spanish Y TERMINO EN ESPANOL: Toward a Typology of Code Switching. Linguistics, 18, 581-618.

Romaine, S. (1995). Bilingualism (2 ${ }^{\text {nd }}$ ed.). UK: Blackwell Publishers.

Shahadujjaman. (2008). Shonkote Jono-shanskriti: Banijjik Dharar ChalachitraShanskritir Beboched. Bangladesh Film Archive Journal, 1, 69-74.

Smith, G. N. (Ed.). (1996). The Oxford History of World Cinema. London: Oxford University Press.

Spolsky, B. (1998). Sociolinguistics. UK: Oxford University Press.

Wardhaugh, R. (1992). An Introduction to Sociolinguistics (2nd ed.). UK: Balckwell Publishers.

\section{Appendix}

\section{Interview Questions}

Instruction: Please answer the following questions in brief.

1. According to you, who are the target audience of Bangladeshi commercial cinema?

2. To what extend do the actors of Bangladeshi cinema use foreign languages other than Bangla?

3. How many types of code switching do you notice in Bangladeshi commercial cinema?

4. Why do the actors use code switching and why is this important?

5. Do you think that the lay people find any problem because of the usage of mix language in cinema? 